\title{
The Integration of Emotion and Reason in Caregiver Pain
}

\section{Assessment}

\section{To the Editor:}

The article by Craig et al $^{1}$ preserves the duality between emotions and reason in the claim that automatic reactions to pain in another are part of a different and evolutionarily earlier system than controlled, reflective responses. This view, which sees emotions as somewhat separate from and in competition with reasoning, is not consistent with clinical observations of growing infants and children. A more promising line taken in the target article is the idea that automatic reactions can be accompanied by controlled responses. This is supported by functional emotional theory, which suggests that emotion and reason are integrated and work together, though at varied levels from one individual to another. ${ }^{10}$

According to Craig et al, ${ }^{1}$ observer response to acute pain in others is a dual process consistent with the automatic (reflexive) and controlled (reflective) reactions of people in pain. Automatic emotional systems likely precede the evolutionary, more recent, controlled reasoning systems, and may correspond to what many neuroscientists describe as the subsymbolic and symbolic systems.5,12 Emotional and cognitive capacities are thought of as separate but related processes. For example, Craig et al $^{1}$ suggest that observer distress to pain in others precedes cognitive efforts to assess what is happening and to plan actions to address the situation.

Damasio ${ }^{4}$ reports that patients with frontal lobe damage retain reasoning abilities but exhibit deficiencies in decision-making based on assessment and appraisal of emotions such as guilt or pride. This view, which preserves the duality between emotions and reason, is not consistent with naturalistic observations of developing infants and young children. For example, Green$\operatorname{span}^{9}$ found that deficits in emotional and symbolic signaling between a child and others undermined the child's ability to distinguish between fantasy and reality, a condition of logical thinking. These individuals revealed problems in coregulated emotional signaling, sequencing, and planning actions, both functions of the

Address reprint requests to Mr. Simon van Rysewyk, University of Tasmania, School of Philosophy, Private Bag 41, Hobart 7001 Australia. E-mail: spvan@postoffice.utas.edu.au

$1526-5900 / \$ 36.00$

(C) 2010 by the American Pain Society

doi:10.1016/j.jpain.2010.02.002 prefrontal cortex. This suggests that the ability to use emotions as signals to probe experience is essential to developing a sense of reality and the capacity to reason. ${ }^{10}$

Clinical work reveals that individual differences in the awareness of emotions and reason and in the ability to integrate them may result from an inability to symbolize emotions. ${ }^{10}$ For example, individuals vary in their ability to signal across the complete range of emotions, and in the ability to name sensations and compare them on reflection with the feelings of others. Greenspan ${ }^{9}$ found that certain individuals who can't fully signal with their emotions tend to discharge intense emotions directly into action (impulsive behavior), somaticize them (headache), or engage in polarized thinking patterns or in fragmented or incomplete reflection. These psychological problems may be associated with a variety of developmental disorders. The dualistic view of an evolutionary recent reasoning system superimposed on the ancient emotional system may therefore confuse the difference between pathological and healthy emotional development.

By contrast, Craig et al ${ }^{1}$ state that emotional and reasoned observer responses to pain in others can operate in parallel. With support from functional emotional theory, this more promising line could claim that certain observer responses in pain assessment may be part of a dynamic system that operates as an integrated whole. Functional emotional theory hypothesizes that individuals who meet age-expected levels of symbolization and emotional signaling can transform intense emotions into complex interactive responses. ${ }^{10}$ In emotionally healthy individuals, intense emotions tend to operate as part of a pattern, where anger or fear coexist as well as levels of symbolization and problem solving. These individuals can exchange emotional signals with others while engaging in symbolic discussions that quickly regulates and modulates emotions. In acute-pain assessment, a well-integrated caregiver confronted by another person in pain can respond by judging why this is happening, or what to do to alleviate the person's pain while limiting personal danger and distress. ${ }^{2}$ This would be a complex response involving problem solving, planning, empathy, and strategic thinking. Alternatively, a less reflective individual might respond to pain in others with intense fear and panic, and discharge his emotions by fleeing from the source of perceived threat. This person would likely experience a conflict between 
van Rysewyk

the fear and conscious control. ${ }^{8}$ Regulated emotions that are part of patterns of interaction are generally linked with more integrated patterns of response and higher levels of reflective thinking. Less regulated emotions tend to be part of aversive situations and less reflective patterns. ${ }^{10,13}$ It could be that the more fully an individual masters emotional signaling, the more flexible his emotional responses in pain assessment and the more these responses function as a highly adaptive instrument of "reason."

Functional emotional theory and developmental psychology can support the idea that emotional signaling links emotion to cognition. In healthy development, catastrophic emotions such as fear and rage are transformed from fixed action responses into emotional signals and interactive emotional patterns in the second half of year 1 and the second year of life and thereafter. $^{9,10}$ As the catastrophic responses of infants to acute pain become shaped by parents and caregivers into emotional signals, the experience of pain can be modulated through emotional interactions with others. $^{3,6,7}$ The perception of pain can be experienced as a freestanding perception or image. ${ }^{10}$ This image can then acquire meaning through additional emotional interactions. For example, the image of pain becomes associated with a whole pattern of emotions: fear, threat, unpleasantness, soothing, comfort, intimacy. As it receives emotional meaning, the image and its associated emotional patterns becomes a symbol. The image of pain is not a single unitary entity based on the interpretation of a physically induced aversive state. It is a representation (a symbolization) of a complex interaction pattern that may involve fear and then comfort, and then less fear and more comfort, and then even less fear and more comfort. What was initially an aversive sensation-pain-is now an interactive emotional pattern that involves the child's emotions and the caregiver's emotions. ${ }^{3,11,13}$ As a growing symbol, it can be combined with other symbols into concepts and ultimately become part of an integrated system of reasoned thinking. To the degree to which caregiver reactions are transformed into signals and interactive patterns that are symbolized, caregiver pain assessment is an integrated response. To the degree that this doesn't happen, automatic emotional responses may remain locked in their primary aversive mode in which perception of pain in others is tied to global action patterns. This pattern would be associated with different types of developmental disorders. ${ }^{10}$
The Journal of Pain 805

Simon van Rysewyk

University of Tasmania

School of Philosophy

Private Bag 41

Hobart 7001 Australia

\section{References}

1. Craig KD, Versloot J, Goubert L, Vervoort T, Crombez G: Perceiving pain in others: Automatic and controlled mechanisms. J Pain 11:101-108, 2009

2. Craig KD: The Social Communication Model of Pain. Canadian Psychology 50:22-32, 2009

3. Craig KD, Korol CT: Developmental issues in understanding, assessing, and managing pediatric pain, in Walco GA, Goldschneider KR (eds): Pain in Children: A Practical Guide for Primary Care, 1st ed. Totowa, NJ, The Humana Press, Inc, 2008, pp 9-20

4. Damasio A: The science of emotion. Keynote address at the NIH, NIMH Conference: Discovering Ourselves: the Science of Emotion, Washington, D.C, Library of Congress, 1998

5. Damasio A: Looking for Spinoza: Joy, Sorrow, and the Feeling Brain, 2nd ed. Orlando, FL, Harcourt, 2003

6. Din L, Riddell RP, Gordner S: Brief Report: Maternal emotional availability and infant-pain-related distress. J Pediatr Psychol 34:722-726, 2009

7. Goodman JE, McGrath PJ: Mothers' modeling influences children's pain during a cold pressor task. Pain 104:559-565, 2009

8. Goubert L, Vervoort T, Sullivan MJL, Verhoeven K, Crombez G: Parental emotional responses to their child's pain: the role of dispositional empathy and parental catastrophizing about their child's pain. Pain 9:272-279, 2008

9. Greenspan I: The growth of the mind and the endangered origins of intelligence, 1st ed. Reading, MA, Addison Wesley, 1997

10. Greenspan I, Shanker SG: The first idea: How symbols, language, and intelligence evolved from our primate ancestors to modern humans, 1st ed. Cambridge, MA, Da Capo Press, 2004

11. Hermann C: Modeling, social learning of pain, in Schmidt RF, Willis WD (eds): The Encyclopedia of Pain, Vol. 1. Heidelberg, Germany, Springer-Verlag, 2007, p 13

12. Le Doux J: Synaptic Self: How Our Brains Become Who We Are, 1st ed. New York, NY, Viking, 2001

13. van Rysewyk S: Comment on: Unconscious affective processing and empathy: An investigation of subliminal priming on the detection of painful facial expressions [Pain 2009; 1-2: 71-75]. Pain 145:364-365, 2009 